\title{
N-Butylpyridoquinoxaline 1, 4-dioxide alleviates experimentally induced leukemia with up-regulating sphingosine-1-phosphate receptors
}

\author{
Mai M. Kabeel, Ahmed M. Ghoneim* \\ Zoology Department, Faculty of Science, Damietta University, Damietta, Egypt.
}

\begin{tabular}{l}
\hline ARTICLE INFO \\
\hline Received on: 15/11/2018 \\
Accepted on: 09/03/2019 \\
Available online: 18/04/2019 \\
\hline Key words: \\
Leukemia, \\
N-Butylpyridoquinoxaline \\
1, 4-dioxide, cytotoxicity, \\
sphingosine-1-phosphate \\
receptors, gene expression.
\end{tabular}

\begin{abstract}
Leukemia, a malignant transformation of hematopoietic cells, is one of the most prevalent types of cancer, especially in children and, globally, it was ranked $10^{\text {th }}$ for cancer incidence and nineth for cancer deaths. Quinoxaline-1, 4-dioxide derivatives have been claimed to have interesting anticancer activities. This study aimed to investigate whether N-Butylpyridoquinoxaline 1, 4-Dioxide (NBPQD) has anti-leukemic effects. Leukemia was stimulated by injecting 7, 12-dimethyl benza[a]anthracene into rats that were then treated with NBPQD. NBPQD successfully restored the normal weight, and the normal total white blood cell (WBC), lymphocyte, and neutrophil counts in the leukemic rats in comparison to either the carrier rat group or the leukemic rats before treating them with NBPQD. Furthermore, NBPQD caused a $71 \%$ reduction in the percentage of blasts in the leukemic rats. Compared to the leukemic animals in which Sphingosine-1-phosphate receptor 1 (S1PR1) and Sphingosine-1-phosphate receptor 5 (S1PR5) were downregulated, NBPQD increased mRNA expression of S1PR1 and S1PR5 in the lymphocytes of leukemic rats 9.6- and 13.3-folds, respectively. Our results indicate that NBPQD is able to alleviate leukemia and it is worthy of further characterization as a potential anti-leukemic drug. Whether up-regulation of S1PR1 and S1PR5 is involved in the mechanism of action of NBPQD needs further investigation.
\end{abstract}

\section{INTRODUCTION}

Leukemia is a malignant transformation of hematopoietic cells which produces an abnormal leukemic clone of cells suppressing the production of normal blood cellular components (Duhrsen and Hossfeld, 1996). Leukemia is one of the most predominant types of cancer, especially in children (Siegel et al., 2018). Being accounting for $2.5 \%$ of all cancers (Forman et al., 2003), leukemia was ranked $11^{\text {th }}$ among the commonly diagnosed types of cancer (Todd et al., 2017).

According to Siegel et al. (2018), around 60,300 new cases of leukemia were expected to be diagnosed in the US in 2018. Based on cell type and rate of growth, leukemia is classified into four main groups: acute lymphocytic, acute myeloid, chronic

\section{"Corresponding Author}

Ahmed M. Ghoneim, Zoology Department, Faculty of Science, Damietta University,Damietta,Egypt.Email:am_ghoneim@du.edu.eg myeloid (CML), and chronic lymphocytic (CLL) (Siegel et al., 2018).

Causes of leukemia are diverse and they could be familial, infectious, physical, or chemical depending on the cell type and the individual variations from one patient to another (Pui, 1995). Obesity, smoking, and exposure to electromagnetic fields and ionizing radiation have been reported to be risk factors for the development of leukemia.

The main approaches to treat leukemia include surgery, radiotherapy, chemotherapy, immunotherapy, and stem cell transplantation (Davis et al., 2014). Leukemia was the first autologous cancer to be cured with chemicals (Aur et al., 1971). Chemotherapy is directed to kill dividing cancer cells because cancer cells attempt to divide when other cells do not; a trait that makes cancer cells susceptible to a broad range of cellular poisons (Pokharel, 2012). Chemotherapeutic drugs include antimetabolites, genotoxic drugs, alkylating agents, intercalating agents, enzyme inhibitors, and spindle inhibitors. 
Quinoxaline-1, 4-dioxide derivatives were found to have interesting anticancer activities, especially in treating solid tumor (Zarranz et al., 2004). Moreover, in the presence of xanthine and xanthine oxidase, quinoxaline derivatives could cleave the DNA helix under hypoxic conditions (Ibrahim and Wally, 2009). Compared to the anticancer agent doxorubicin, N-Butylpyridoquinoxaline 1, 4-Dioxide (NBPQD) showed higher activity on the human liver cell line HEPG2 cells (Habib et al., 2012). NBPQD has been reported to have a high cytotoxicity against Ehrlich ascites carcinoma (EAC) cells in vitro (Habib et al., 2012).

The main side effect of chemotherapy is myelosuppression and anemia. Treatment of EAC-bearing mice with NBPQD did not cause such myelo-suppression and anemia. This compound was reported to restore the level of hemoglobin and red blood cell (RBC) count close to their normal values (Habib et al., 2012; Subramanian and Gowry, 2011). NBPQD is thought to possess a protective action for the hematopoietic system because of its ability to scavenge free radicals through superoxide dismutase-like activity (Habib et al., 2012). The present study was thus designed to explore whether NBPQD has any anti-leukemic effects.

Sphingosine-1-phosphate receptors (S1PRs) are a family of plasma membrane G-protein-coupled receptors (GPCRs) that play fundamental roles in cell survival, proliferation, migration, and differentiation during the development and progression of cancer, including hematological malignancies, which make them critical and important targets for potential therapeutics for cancers (Watters et al., 2011). In addition, GPCRs are considered ideal targets for drug development due to the specificity of chemical substances that can be generated (Watters et al., 2011). To investigate the roles of S1PRs in the development of leukemia and to identify their potential agonists and antagonists, we evaluated the expression of S1PR1 and S1PR5.

\section{ANIMALS AND METHODS}

\section{Animals}

Twenty-five male albino Wistar rats (65-75 g) were maintained in rearing cages in the animal house of our institute. Animals were divided into five groups, five rats each. Carrier group: rats were injected in the caudal vein with Dimethyl sulfoxide (DMSO) every 2 weeks (four times in total). 7, 12-dimethyl benza[a]anthracene (DMBA) group: animals were injected in the caudal vein with DMBA every 2 weeks (four times in total). DMBA + NBPQD group: animals were injected in the caudal vein every 2 weeks with DMBA (four times in total) and the blood was collected from the caudal vein ("DMBA before NBPQD" subgroup). This subgroup of animals was then injected intra-peritoneally with NBPQD daily for 14 days ("NBPQD after DMBA" subgroup). NBPQD group: rats were injected intraperitoneally with NBPQD daily for 14 days. Untreated group: animals were fed on normal diet and drinking water for 14 days.

\section{Induction of leukemia, growth performance, and WBCs count}

Leukemia was experimentally induced and assessed in rats, and the evaluation of growth performance and WBC count were performed as previously mentioned (Kabeel et al., 2018).

\section{Quantitation of gene expression}

Blood lymphocytes were collected from three rats of each of the studied groups by Lymphocyte Separation Medium (\#17$829 \mathrm{E}$, Lonza) following the recommended instructions. Total RNA was isolated from the collected cells by GeneJET RNA Purification Kit (\#K0731, Thermo Scientific). Pure RNA was converted into cDNA by RevertAid H Minus cDNA Synthesis Kit (\#K1631, Thermo Scientific) following the recommended instructions. SYBR Green PCR Mix (\#K0251, Thermo Scientific) was used to perform Quantitative Real Time PCR for S1PR1 and S1PR5 in $20 \mu 1$ scale reactions. cDNAs of S1PR1 and S1PR5 were quantitated using the primers: S1PR1 sense: 5'-CCGCTTGAGCGAGGCTGCTG-3', S1PR1 antisense: 5'-CTATGATATCATAGTTGCCATAGTC-3', S1PR5 sense: 5'-TCTAGAGCGCCACCTTACCATG-3', and the antisense primer: 5'- AAATCCTTGCATAGAGCGCACAG-3'. $\beta$-actin was detected as the reference gene using the sense primer: 5'-CCTTCTACAAATGAGCTGCGT-3' and the antisense primer: 5'-CCTGGATAGCAACGTACATG-3'. Reactions were cycled in Mx3000P QPCR Systems (Stratagene) at $95^{\circ} \mathrm{C}$ for 10 minutes, followed by cycling 40 times at $95^{\circ} \mathrm{C}$ for 15 seconds, $60^{\circ} \mathrm{C}$ for 15 seconds, and $72^{\circ} \mathrm{C}$ for 30 seconds. Data analysis was performed by the $\Delta \Delta \mathrm{Ct}$ method.

\section{Statistical analysis}

All values in the text and figures were presented as arithmetic means \pm standard error of the mean. Statistical significance was determined by one-way analysis of variance with Tukey's HSD test and $p$ values $\leq 0.05$ were considered significant.

\section{RESULTS}

\section{NBPQD alleviates leukemia in rats}

To determine whether NBPQD has an anti-leukemic effect, leukemia was stimulated in rats by DMBA injection followed by intra-peritoneal treatment of rats with NBPQD. In comparison to "carrier" group, there was a significant decrease $(p=0.040)$ in the weight gain in the leukemic "DMBA before NBPQD" subgroup. After applying NBPQD on the leukemic (DMBA-treated) rats, no significant change $(p=0.612)$ in the weight gain was detected between "NBPQD after DMBA" and the "carrier" groups (Fig. 1). This reveals the recovery of weight in the leukemic rats injected with NBPQD. A significant rise ( $p$ $=0.000)$ in the total WBC number was noticed in the leukemic "DMBA before NBPQD" subgroup in comparison to the "carrier" animal group (Fig. 2). After applying NBPQD on the leukemic (DMBA-treated) rats, no significant change $(p=0.985)$ in the total WBC number was noticed between the leukemic "NBPQD after DMBA" subgroup and the "carrier" group, revealing recovery of the normal total WBC number in the NBPQD-treated rats (Fig. $2)$. Within the same animal group, a significant rise $(p=0.001)$ in the weight gain and a significant decrease $(p=0.008)$ in the total WBC number were noticed in the DMBA-treated rats after applying NBPQD ("NBPQD after DMBA" subgroup) compared to DMBA-treated rats before applying NBPQD ("DMBA before NBPQD" subgroup), confirming restoration of the normal weight gain and total count of WBCs after treating rats with NBPQD (Figs. 1 and 2). 


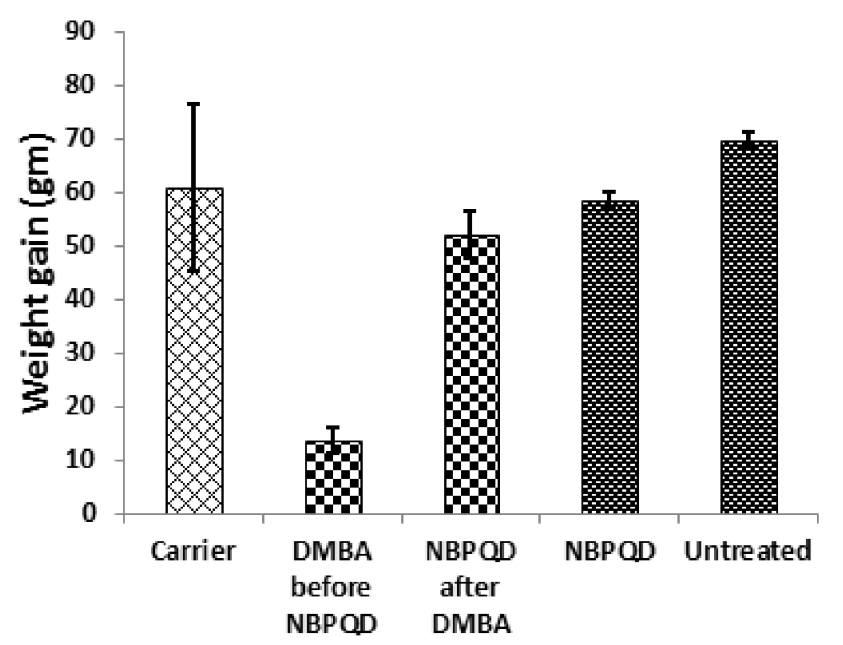

Figure 1. Weight gain in the "Carrier," "DMBA before NBPQD," "NBPQD after DMBA," "NBPQD," and "Untreated" groups. NBPQD recovered normal weight in the leukemic rats and it had no significant effect on the untreated rats.

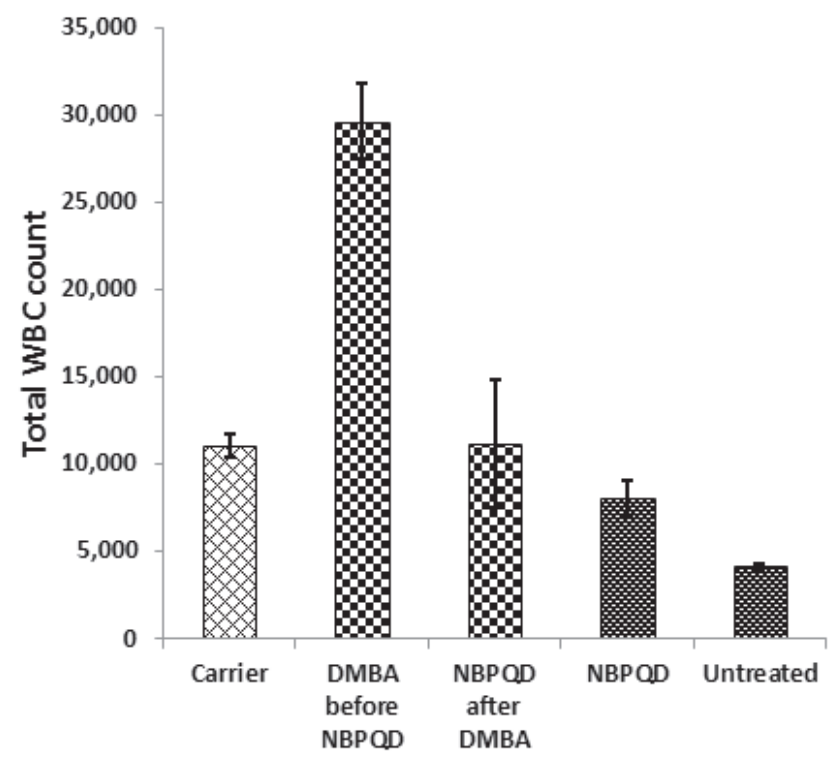

Figure 2. Total WBC count in the "Carrier," "DMBA before NBPQD," "NBPQD after DMBA," "NBPQD," and "Untreated" groups. NBPQD restored the normal WBC in the leukemic rats and it had no significant effect on the untreated rats.

In comparison to the "carrier" group, a significant decrease $(p=0.002)$ in the lymphocytes $\%$ was noticed in the leukemic "DMBA before NBPQD" subgroup of animals (Fig. 3). After applying NBPQD on the leukemic (DMBA-treated) rats, no significant change $(p=0.255)$ was noticed in the lymphocyte $\%$ between "NBPQD after DMBA" and the "carrier" animal groups, revealing the recovery of the normal number of lymphocytes after treatment with NBPQD (Fig. 3). A significant reduction ( $p=$ 0.043 ) in the neutrophil \% was noticed in the leukemic "DMBA before NBPQD" subgroup in comparison to the "carrier" animal group (Fig. 4). After applying NBPQD on the leukemic (DMBAtreated) animals, there was no significant change $(p=0.363)$ in

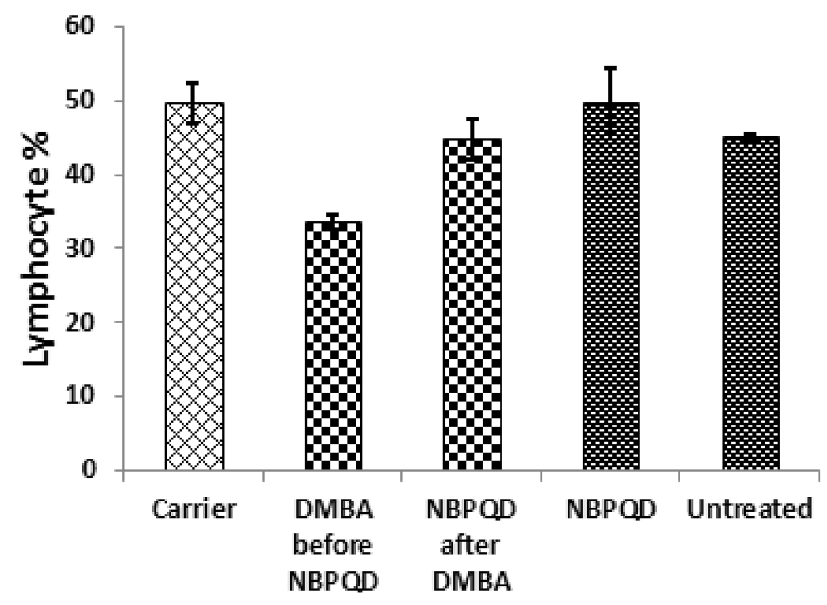

Figure 3. Lymphocyte percentage in the "Carrier," "DMBA before NBPQD," "NBPQD after DMBA," "NBPQD," and "Untreated" groups. NBPQD restored the normal lymphocyte percentage in the leukemic rats and it had no significant effect on the untreated rats.

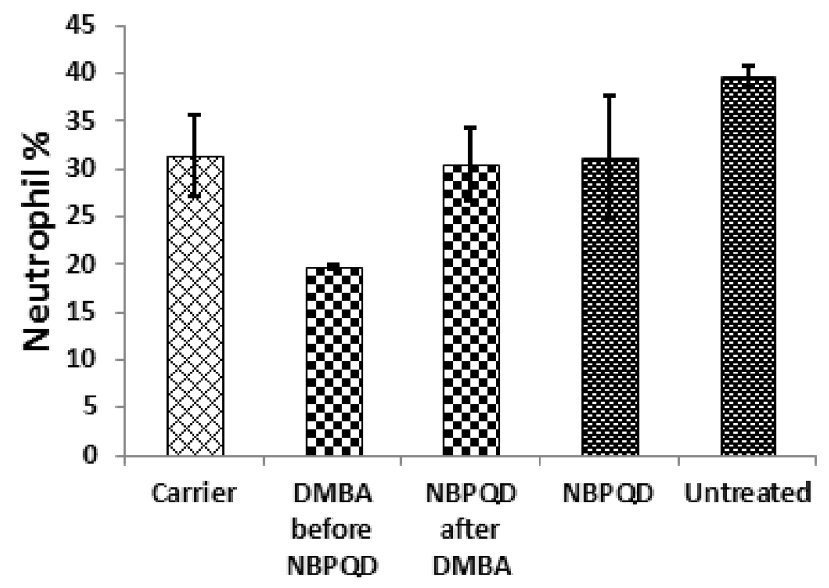

Figure 4. Neutrophil percentage in the "Carrier," "DMBA before NBPQD," "NBPQD after DMBA," "NBPQD," and "Untreated" groups. NBPQD restored the normal neutrophil percentage in the leukemic rats and it had no significant effect on the untreated rats.

the neutrophil \% between the "NBPQD after DMBA" and the "carrier" groups, revealing an increase in the neutrophils \% after treating rats with NBPQD (Fig. 4). A statistically significant increase $(p=0.000)$ in the blast $\%$ was recorded in the leukemic "DMBA before NBPQD" subgroup in comparison to the "carrier" group (Fig. 5). With the application of NBPQD on the leukemic (DMBA-treated) rats, $71 \%$ reduction in the blast \% was noticed (Fig. 5). Thus, treating the leukemic rats with NBPQD resulted in a very considerable decrease in the percentage of blasts.

Inside the same treated group, a significant increase $(p=$ $0.010)$ in lymphocyte \% was noticed in the "NBPQD after DMBA" subgroup in comparison to the leukemic "DMBA before NBPQD" subgroup, revealing recovery of the normal number of lymphocytes after treating animals with NBPQD (Fig. 3). A noticeable but nonsignificant rise $(p=0.165)$ in the neutrophil \% was recorded in the "NBPQD after DMBA" subgroup in comparison to the leukemic 


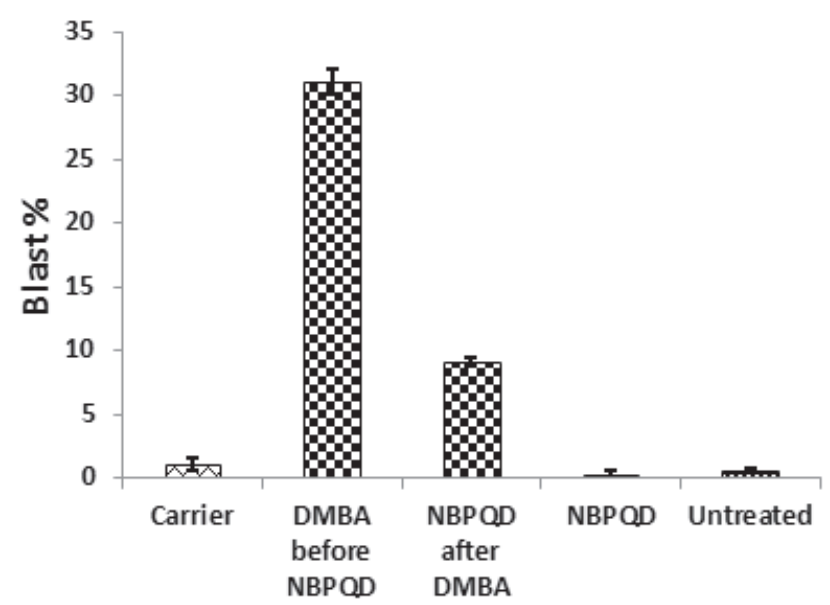

Figure 5. Blast percentage in the "Carrier," "DMBA before NBPQD," "NBPQD after DMBA," "NBPQD," and "Untreated" groups. NBPQD caused a remarkable reduction on the blast $\%$ in the leukemic rats and it had no significant effect on the untreated rats.

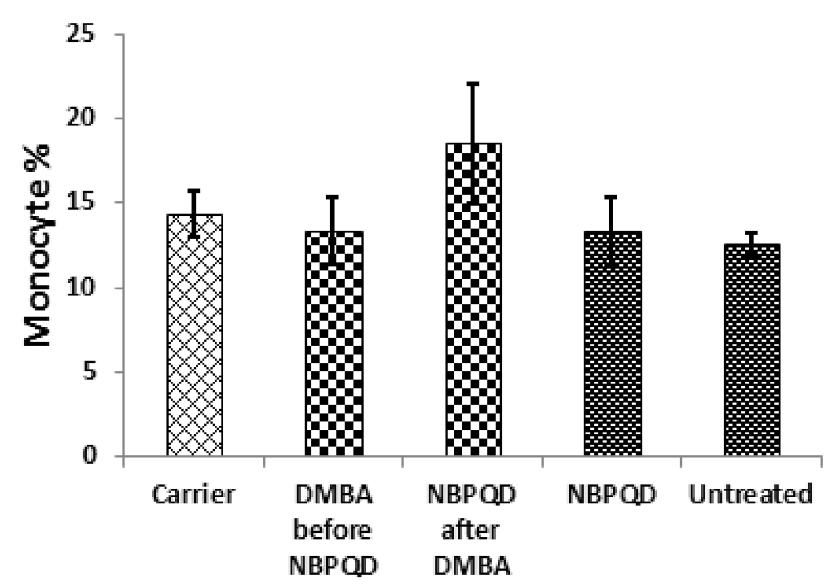

Figure 6. Monocyte percentage in the "Carrier," "DMBA before NBPQD," "NBPQD after DMBA," "NBPQD," and "Untreated" groups. NBPQD had no significant effect on the monocyte percentage in the leukemic rats or the untreated rats.

"DMBA before NBPQD" subgroup (Fig. 4). A statistically significant reduction $(p=0.000)$ in the blast \% was noticed in the "NBPQD after DMBA" subgroup in comparison to the leukemic "DMBA before NBPQD" subgroup, confirming the decrease in the number of blasts after treating rats with NBPQD (Fig. 5).

No significant change in the percentage of monocytes $(p=0.703)$, eosinophils $(p=0.160)$, or basophils $(p=0.630)$ between the "carrier" group and the "DMBA before NBPQD" subgroup (Figs. 6-8). After applying NBPQD on the leukemic (DMBA-treated) animals, no significant change in the percentage of monocytes $(p=0.262)$, eosinophils $(p=0.100)$, or basophils ( $p$ $=0.943$ ) was detected between the "carrier" group and "NBPQD after DMBA" subgroup (Figs. 6-8). Inside the same treated group, no significant change in the percentage of monocytes $(p=0.247)$, eosinophils $(p=0.080)$, or basophils $(p=0.774)$ was detected between the "NBPQD after DMBA" and the leukemic "DMBA before NBPQD" subgroups (Figs. 6-8).

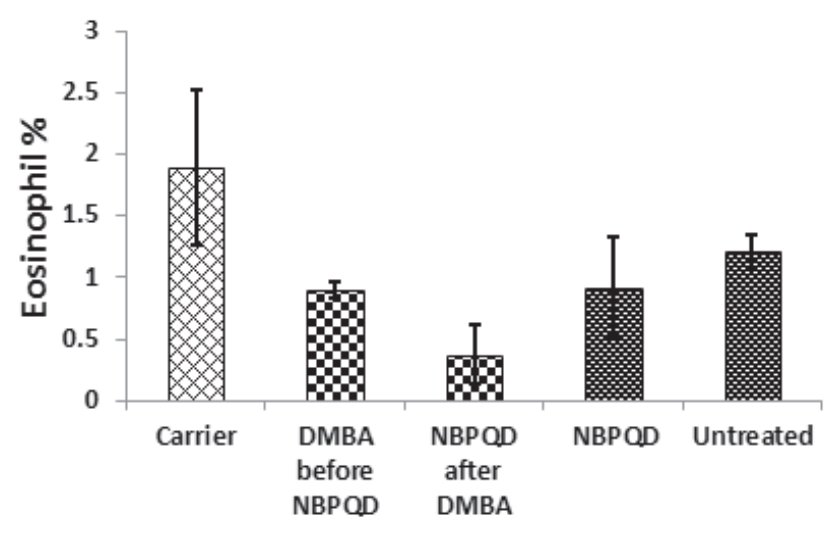

Figure 7. Eosinophil percentage in the "Carrier," "DMBA before NBPQD," "NBPQD after DMBA," "NBPQD," and "Untreated" groups. NBPQD had no significant effect on the eosinophil percentage in the leukemic rats or the untreated rats.

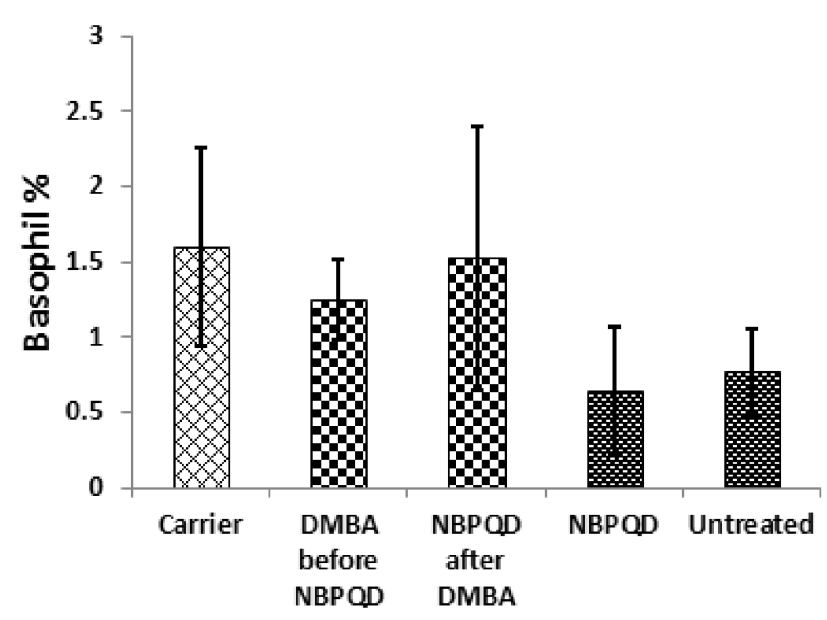

Figure 8. Basophil percentage in the "Carrier," "DMBA before NBPQD," "NBPQD after DMBA," "NBPQD," and "Untreated" groups. NBPQD had no significant effect on the basophil percentage in the leukemic rats or the untreated rats.

\section{Effect of NBPQD on the untreated rats}

To examine the effect of NBPQD on the untreated rats, rats were intra-peritoneally injected with NBPQD for 14 days and compared to the "untreated" group with respect to the studied biological parameters. No significant differences between the "untreated" and the "NBPQD" groups in weight gain ( $p=$ $0.623)$, total WBC number $(p=0.093)$, lymphocyte $\%(p=0.621)$, neutrophil $\%(p=0.117)$ and the percentage of blasts $(p=0.403)$, monocytes $(p=0.807)$, eosinophils $(p=0.389)$, and basophils $(p$ $=0.754)($ Figs. 1-8).

\section{S1PR1 and S1PR5 are involved in alleviating leukemia by NBPQD}

To investigate whether alleviation of leukemia by NBPQD involves regulating the expression of S1PR1 and S1PR5, lymphocytes were isolated from the "untreated," "carrier," "DMBA," "DMBA before NBPQD," "NBPQD after DMBA," and "NBPQD" groups, and mRNA expression of S1PR1 and 


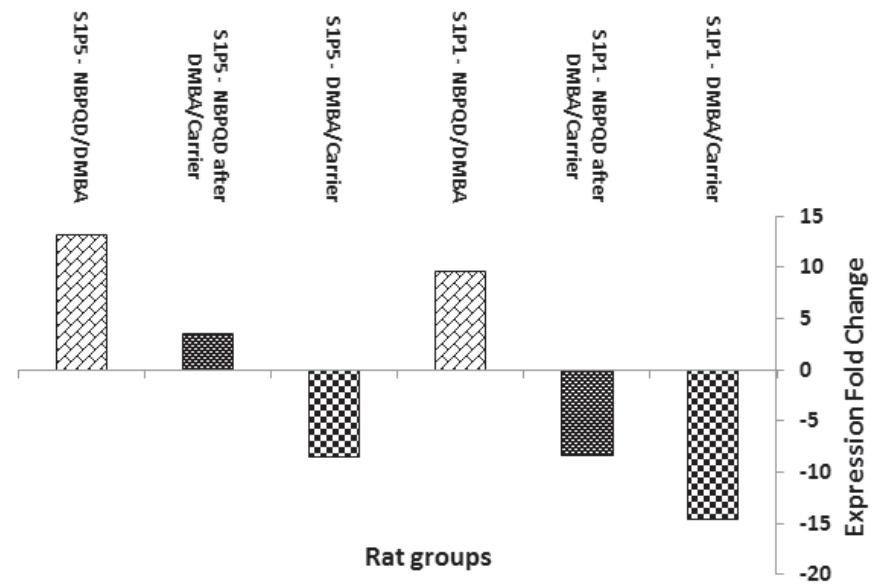

Figure 9. Fold change of S1PR1 and S1PR5 expression in the animal groups: DMBA (in comparison to carrier), NBPQD after DMBA (in comparison to carrier), and NBPQD (in comparison to DMBA). mRNA expression of S1PR1 and S1PR5 was considerably downregulated in the leukemic rats and NBPQD upregulated their expression 9.6- and 13.3-folds, respectively.

S1PR5 was evaluated by real-time PCR in three animals of each studied group. The fold change of S1PR1 and S1PR5 expression was evaluated by $\Delta \Delta \mathrm{Ct}$ method and the "log" of fold change was presented in Figure 9.

In comparison to the "carrier" group, S1PR1 mRNA expression was reduced 14.6 times in the leukemic "DMBA" group. The reduction in S1PR1 expression was ameliorated in the "NBPQD after DMBA" group to 8.2 times in comparison to the "carrier" group. In comparison to the leukemic "DMBA" group, NBPQD increased S1PR1 expression 9.6-folds (Fig. 9).

Compared to the "carrier" group, S1PR5 mRNA expression decreased 8.6 times in the leukemic "DMBA" group. The reduction in S1PR5 expression was abolished and S1PR5 was overexpressed in the "NBPQD after DMBA" group to 3.6 times in comparison to the "carrier" group. In comparison to the leukemic "DMBA" group, NBPQD increased S1PR1 expression 13.3-folds (Fig. 9).

\section{DISCUSSION}

In this study, we have shown that NBPQD is able to alleviate experimentally induced leukemia in rats by recovering the normal body weight in addition to recovering the normal numbers of total WBCs, lymphocytes, and neutrophils. Importantly, NBPQD caused $71 \%$ reduction in the blast percentage in the treated leukemic rats. In addition, NBPQD upregulated S1PR1 and S1PR5 expression several folds and, therefore, it can be considered as potential S1PR1 and S1PR5 agonist.

DMBA induces leukemia well in experimental animals (Kabeel et al., 2018; Sugiyama et al., 2002) because of its ability to produce DMBA-DNA adducts that stimulate a highly specific mutation at the second position in the codon number 61 of $N$-ras gene (Osaka et al., 1995). The experimental $N$-ras gene mutation is similar to that in human leukemias and is induced 48 hours after DMBA treatment (Sugiyama et al., 2002). Like other Ras gene mutations, this mutation is believed to drive a variety of signaling pathways causing cell proliferation (Maruta and Burgess, 1994). Also, DMBA generates too much reactive oxygen species during its metabolism, which results in lipid peroxidation, damage in DNA, and exhaustion of the antioxidant defense systems in the cell
(Bharali et al., 2003; Kumar et al., 2014). According to Huggins and Sugiyama (1966), the induced leukemia is a mixture of diffuse hepatic leukemia of erythroblastic stem cells, and myelogenous, lymphoblastic, and thymic leukemias.

In the current study, weight loss has been observed in the DMBA-induced leukemia. Previous studies showed that leukemia causes weight loss due to eating less, using more energy, high metabolism, or hyperthyroidism (Huggins and Sugiyama, 1966; Sessions, 2007).

In the present study, a significant increase in total WBC count and a highly significant increase in the blast percentage were recorded in the leukemic DMBA-treated animals. These results are similar to the results of Huggins and Sugiyama (1966) and N'jai et al. (2010) who found that the administration of DMBA increased the total WBC count. The presence of blasts is the most significant observation in the leukemic peripheral film. DMBA impairs maturation of myeloid and lymphoid progenitor cells because its metabolites are able to inhibit proliferation or differentiation of hematopoietic stem cells (N'Jai et al., 2010).

In the present study, DMBA induced a decrease in lymphocyte and neutrophil percentages. Previous studies suggested that DMBA targets selectively WBCs rather than red cells or thrombocyte precursors in the bone marrow because it did not cause any significant changes in red blood cell numbers, hematocrit value, or platelet numbers, but it produced a reduction in numbers of neutrophils and lymphocytes (El-Bakry et al., 2013; N'Jai et al., 2010).

Current results show that the application of NBPQD efficiently recovered the weight loss in the leukemic rats. Administration of NBPQD for 14 consecutive days, in this study, successfully restored the normal total WBC number and the normal lymphocyte and neutrophil percentages in the leukemic animals in comparison to either the carrier group or the group of leukemic rats before applying NBPQD. Moreover, NBPQD caused $71 \%$ decrease in the blast percentage in the leukemic rats.

Previous studies demonstrated some anticancer activities for NBPQD (Zarranz et al., 2004). Habib et al. (2012) demonstrated that treatment with NBPQD induced an increase in the proliferation of all types of leukocytes, and, therefore, it was proposed that NBPQD stimulates the early steps of both myeloid and lymphoid maturation pathways of leukocytes.

The observation that NBPQD also caused a decrease in the percentage of the DMBA-induced blasts in this study may indicate that NBPQD possesses a protective action for the hemopoietic system. This supports the finding of Habib et al. (2012) that the anti-tumor potential of NBPQD was because of its superoxide dismutase-like activity, which scavenges the free radicals.

In general, quinoxaline derivatives have a broad variety of biological activities, including antimicrobial, anti-inflammatory, antioxidant, anticancer, and antihistaminic activities (Ganapathy et al., 2007). Specific quinoxaline derivatives showed selective cytotoxicity against hypoxic cells in solid tumors (Gali-Muhtasib et al., 2001).

Quinoxaline-1, 4-dioxide derivatives were reported to possess anticancer activities especially in the treatment of solid tumor (Zarranz et al., 2004). Treatment of EAC-bearing mice with NBPQD brought the hemoglobin level and RBC count back near to their normal values, and, therefore, it overcomes anemia and myelo-suppression which are the major problems of 
chemotherapy (Subramanian and Gowry, 2011). This indicates that NBPQD protects the hematopoietic system because of its ability to scavenge the free radicals (Habib et al., 2012).

NBPQD prolonged the life span of the animal model (Habib et al., 2012), thus increasing the potentiality that NBPQD has a remarkable anti-tumor activity in experimental animals (Habib et al., 2012). Prolongation of the animal's life span is a reliable criterion for evaluating the antitumor capability of any agent (Gupta et al., 2004).

According to Habib et al. (2012), the effect of NBPQD on the tumor model was thought to depend on: (1) the ability to cleave the DNA under hypoxic conditions in the presence of xanthine and xanthine oxidase (Ibrahim and Wally, 2009), (2) the breaks that take place in the DNA double helix when NBPQD is introduced to tumor cells (Unak and Unak, 1996), (3) the ability of NBPQD to produce oxygen radicals that can attack tumor cells, where, tumor cells face a reduction in the antioxidants system (Ibrahim and Wally, 2009), or (4) possible cooperation of these three mechanisms to produce this effect.

To the best of our knowledge, this study is the first report that shows the effects of NBPQD on DMBA-induced leukemia. Data presented here indicated that NBPQD given to leukemic rats for 14 consecutive days was able to alleviate the leukemic effects and restore the normal levels of many biological parameters.

Our results indicated that S1PR1 expression at the mRNA level in lymphocytes decreased 14.6 times in the leukemic rats. This decrease in S1PR1 expression in the leukemic rats has been ameliorated to 8.2 folds after treating rats with NBPQD. In other words, compared to the leukemic animals, NBPQD was able to increase S1PR1 expression 9.6 folds.

Levels of S1PR1 and S1PR5 mRNAs are considered correlating with their protein levels. In a few types of cells, such correlation may be weak; however, all studies on S1PR1 and S1PR5 in the different types of cells, there was no controversy between their mRNA expression and their protein levels.

S1P and S1PR1 play an essential role in regulating the exit of $T$ and $B$ cells from peripheral lymphoid organs toward blood and lymph. The levels of S1PR1 determine the transit time of normal lymphocytes through lymph nodes. Surface S1PR1 is very sensitive to the presence of S1P, as once lymphocytes get into circulation; the expression of S1PR1 gene on the cell surface is promptly down-regulated (Lo et al., 2005) by liganddependent receptor internalization as a consequence of the higher S1P levels in blood and lymph (Pappu et al., 2007). One reason for why lymphocytes may have to downregulate S1PR1 after entering the bloodstream is to be able to overcome the attractive pulling of S1P and moving back into lymphoid tissue (Arnon et al., 2014).

S1PR1 gene is expressed in a multitude of hematological malignancies, including classical Hodgkin's lymphoma, B-chronic lymphocytic leukemia, activated B cell-like diffuse large B cell lymphoma (Capitani et al., 2012; Kluk et al., 2013). The expression of S1PR1 is reduced by different signals that leukemic cells may encounter within the niche of lymphoid tissues and this delays their egress to the circulation and, thus, extends their stay at survival micro-environments (Borge et al., 2014).

S1PR1 is abundantly expressed on small lymphocytes (Nishimura et al., 2010) and S1P receptors levels are regulated during trafficking of lymphocyte and setting their dwell time in the peripheral lymphoid organs (Capitani et al., 2012).

Compared with normal B cells, S1PR1 gene expression is reduced in CLL (Borge et al., 2014; Capitani et al., 2012). S1PR1 gene expression is regulated by B Cell Receptor (BCR) signaling. $\mathrm{BCR}$ signaling represses S1PR1 expression and function on CLL cells, so, the egression from the lymphoid tissues is delayed and the retention of CLL cells in lymph nodes occurs (Capitani et al., 2012; Till et al., 2015). Treatment with BCR signaling inhibitors relieved this BCR facilitated repression of S1PR1 expression and resulted in the exit of CLL cells from affected lymph nodes (Krysov et al., 2010). In vivo treatment using Fostamatinib inhibited BCR signaling, cellular activation, and tumor proliferation in patients with relapsed CLL (Herman et al., 2010).

S1PR1 gene was identified as a reactive oxygen speciesresponsive gene in CLL and the supportive micro-environment signals reduced S1PR1 gene expression by modifying the redox status of the cells (Capitani et al., 2012).

Results of this sudy indicated 8.6 times reduction in S1PR5 mRNA expression in lymphocytes in the leukemic animals in comparison to the control animal group. This reduction in S1PR5 expression in the leukemic rats has been abolished and S1PR5 was overexpressed 3.6-folds after treating rats with NBPQD. In other words, compared to the leukemic rats, NBPQD was able to upregulate S1PR5 expression 13.3-folds.

S1PR5 gene is expressed in brain and spleen, and it was first discovered in peripheral blood mononuclear cells from $\mathrm{T}$ celllarge granular lymphocyte leukemia (T-LGL) (Kothapalli et al., 2002). S1PR5 is upregulated in $\sim 80 \%$ of T-LGL leukemia patients (Shah et al., 2008). S1PR5 is the predominant type of S1P receptor expressed on normal natural killer (NK) cells and it is involved in the egress of NK cells from the bone marrow to the blood (Jenne et al., 2009; Walzer et al., 2007).

S1PR5 decreases the activity of adenylyl cyclase and extracellular signal-regulated kinases in many cell lines in the absence of S1P, and, thus, it induces cell detachment and rounding without enhancing apoptosis (Niedernberg et al., 2003). This effect could contribute to or even prompt cell migration by preventing strong attachment to the bone marrow stromal substrate (Debien et al., 2013).

Many agonists and antagonists which target S1PR1 were produced and are under clinical testing (Kunkel et al., 2013). FTY720, approved by the FDA and used as an oral therapy for treating diseases (Chun and Brinkmann, 2011), is a sphingosine analog that is phosphorylated in the cells and acts as a powerful agonist for most of sphingosine-1-phosphate receptors (Jeffery et al., 2011).

FTY720 has displayed anti-tumor activity by prompting apoptosis of solid tumor cells or leukemic cells through S1PRdependent or independent mechanisms (Jeffery et al., 2011; Zhang et al., 2010). FTY720 induced apoptosis of LGL cells and sensitized these cells to Fas-mediated cell death (Shah et al., 2008) and it, also, inhibited CML stem cell proliferation and expansion in vitro (Neviani et al., 2013). Several data support FTY720 for anticancer therapy in various cancer settings (Ogretmen, 2018).

Because S1PR1 and S1PR5 are upregulated by NBPQD treatment, it is possible that NBPQD acts as an agonist that regulates the expression of these receptors and controls the WBC and lymphocyte number in the treated leukemic rats. It is noteworthy to 
mention that as members of the GPCR family, S1P receptors can be considered good targets for drug development due to the specificity of the chemical substances that can target them.

\section{CONCLUSION}

This study determined the ability of NBPQD to alleviate experimentally induced leukemia in rats. NBPQD restored the weight loss, the normal total WBC, lymphocyte and neutrophil counts, and most importantly, it caused $71 \%$ reduction in the blast percentage in the treated leukemic rats. S1PR1 and S1PR5 were downregulated in the leukemic rats and NBPQD ameliorated this down regulation of S1PR1 and S1PR5. NBPQD up-regulated S1PR1 and S1PR5 expression 9.6- and 13.3-folds, respectively. It is possible that S1PR1 and S1PR5 are involved in the mechanism of the anti-leukemic action of NBPQD. Further characterization of the pathways initiated by the NBPQD as a promising anti-leukemic agent is necessary.

\section{AUTHORS' CONTRIBUTIONS}

Mai M. Kabeel has done experiments and prepared the initial draft of this manuscript and Ahmed M. Ghoneim has made the experimental design, participated in doing experiments, and revised the manuscript.

\section{ACKNOWLEDGEMENTS}

Authors would like to thank Heba M. Waly, Chemistry Department in our institute, for providing NBPQD and Radwa R. El-Tahan, Zoology Department in our institute, for her help during this study.

\section{FINANCIAL SUPPORT AND SPONSORSHIP}

This study was funded in part by Zoology Department, faculty of Science, Damietta University, Egypt.

\section{CONFLICT OF INTERESTS}

The authors declare that they have no conflict of interest.

\section{ETHICAL APPROVAL}

All animals in the current study were reared and manipulated as approved by our faculty (No. 5/2/2/3) and in accordance with the National Institute of Health guide for the care and use of laboratory animals.

\section{REFERENCES}

Arnon TI, Cyster JG. Blood, sphingosine-1-phosphate and lymphocyte migration dynamics in the spleen. Curr Top Microbiol Immunol, 2014; 378:107-28.

Aur RJA, Simone JV, Hustu HO, Walters T, Borella L, Pratt C, Pinkel D. Central nervous system therapy and combination chemotherapy of childhood lymphocytic leukemia. Blood, 1971; 37:272-81.

Bharali R, Tabassum J, Azad MRH. Chemomodulatory effect of Moringa oleifera, lam, on hepatic carcinogen metabolising enzymes, antioxidant parameters and skin papillomagenesis in mice. Asian Pac J Cancer Prev, 2003; 4:131-9.

Borge M, Lenicov F, Nannini PR, de los Ríos Alicandú MM, Podaza E, Ceballos A, Grecco H, Cabrejo M, Bezares RF, Morande PE, Oppezzo P, Giordano M, Gamberale R. The expression of sphingosine-1 phosphate receptor- 1 in chronic lymphocytic leukemia cells is impaired by tumor microenvironmental signals and enhanced by piceatannol and R406. J Immunol, 2014; 193:3165-74.
Capitani N, Patrussi L, Trentin L, Lucherini OM, Cannizzaro E, Migliaccio E, Frezzato F, Gattazzo C, Forconi F, Pelicci P, Semenzato G, Baldari CT. S1PR1 expression is controlled by the pro-oxidant activity of p66Shc and is impaired in B-CLL patients with unfavorable prognosis. Blood, 2012; 120:4391-9.

Chun J, Brinkmann V. A mechanistically novel, first oral therapy for multiple sclerosis: the development of fingolimod (FTY720, Gilenya) Discov Med, 2011; 12:213-28.

Davis AS, Viera AJ, Mead MD. Leukemia: an overview for primary care. Am Fam Physician, 2014; 89:731-8.

Debien E, Mayol K, Biajoux V, Daussy C, De Aguero MG, Taillardet M, Dagany N, Brinza L, Henry T, Dubois B, Kaiserlian D, Marvel J, Balabanian K, Walzer T. S1PR5 is pivotal for the homeostasis of patrolling Monocytes. Eur J Immunol, 2013; 43:1667-75.

Duhrsen U, Hossfeld DK. Stromal abnormalities in neoplastic bone marrow diseases. Ann Hematol, 1996; 73:53-70.

El-Bakry HA, Abd-Elghany MI, Soliman SS. Preventive effect of Commiphora molmol on rat mammary carcinogenesis induced by 7 , 12-dimethylbenz(A)Anthracene (DMBA) in comparison with melatonin. Global J Pharmacol, 2013; 7:398-411.

Forman D, Stockton D, Møller H, Quinn M, Babb P, De Angelis $\mathrm{R}$, Micheli A. Cancer prevalence in the UK: results from the EUROPREVAL study. Ann Oncol, 2003; 14:648-54.

Gali-Muhtasib HU, Haddadin MJ, Rahhal DN, Younes IH Quinoxaline 1,4-dioxides as anticancer and hypoxia-selective drugs. Oncol Rep, 2001; 8:679-84.

Ganapathy S, Ramalingam P, Babu Rao CH. Antibacterial, antifungal and antitubercular screening of some novel condensed bridgehead nitrogen heterocycles of quinoxalines. Ind J Heterocyclic Chem, 2007; 16:283-86.

Gupta A, Mazumder UK, Kumar RS, Kumar TS. Anti-tumor activity and anti-oxident role of Bauhinia racemosa against Ehrlich ascites carcinoma in Swiss albino mice. Acta Pharmacol Sinica, 2004; 25:1070-6.

Habib SA, Ibrahim IT, Abd-Eldayem MA, El-Sheshtawey MM, Waly HM. N-Butylpyridoquinoxaline 1,4-dioxide (NBPQD) as a new potent for tumor imaging and therapy. Nat Sci, 2012; 4:1074-84.

Herman SEM, Gordon AL, Wagner AJ, Heerema NA, Zhao W, Flynn JM, Jones J, Andritsos L, Puri KD, Lannutti BJ, Giese NA, Zhang X, Wei L, Byrd JC, Johnson AJ. Phosphatidylinositol 3-kinase-d inhibitor CAL-101 shows promising preclinical activity in chronic lymphocytic leukemia by antagonizing intrinsic and extrinsic cellular survival signals Blood, 2010; 116:2078-88.

Huggins CB, Sugiyama T. Induction of leukemia in rat by pulse doses of 7,12-dimethylbenz(a)anthracene. Proc Natl Acad Sci USA, 1966; 55:74-81.

Ibrahim IT, Wally MA. Synthesis, labeling and biodistribution of 99mTc-3-amino-2-quinoxalin-carbonitrile 1,4-dioxide in tumor bearing mice. J Radioanal Nucl Chem, 2009; 285:169-75.

Jeffery DR, Markowitz CE, Reder AT, Weinstock-Guttman B, Tobias K. Fingolimod for the treatment of relapsing multiple sclerosis Expert Rev Neurother, 2011; 11:165-83.

Jenne CN, Enders A, Rivera R, Watson SR, Bankovich AJ, Pereira JP, Xu Y, Roots CM, Beilke JN, Banerjee A, Reiner SL, Miller SA, Weinmann AS, Goodnow CC, Lanier LL, Cyster JG, Chun J. T-betdependent S1PR5 expression in NK cells promotes egress from lymph nodes and bone marrow. J Exp Med, 2009; 206:2469-81.

Kabeel MM, Ghoneim AM, Mansy SE. Anti-leukemic activity of a four-plant mixture in a leukemic rat model. JoBAZ, 2018; 79:7.

Kluk MJ, Ryan KP, Wang B, Zhang G, Rodig SJ, Sanchez T Sphingosine-1-phosphate receptor 1 in classical Hodgkin lymphoma: assessment of expression and role in cell migration. Lab Invest, 2013; 93:462-71.

Kothapalli R, Kusmartseva I, Loughran TP. Characterization of a human sphingosine-1-phosphate receptor gene (S1PR5) and its differential expression in LGL leukemia. Biochim Biophys Acta, 2002; 1579:117-23. 
Krysov S, Potter KN, Mockridge CI, Coelho V, Wheatley I, Packham G, Stevenson FK. Surface IgM of CLL cells displays unusual glycans indicative of engagement of antigen in vivo. Blood, 2010; 115:4198-205.

Kumar R, Kaur R, Singh AP, Arora S. Diminution of hepatic response to 7, 12-dimethylbenz $(\alpha)$ anthracene by ethylacetate fraction of Acacia catechu Willd. through modulation of xenobiotic and anti-oxidative enzymes in rats. PLoS One, 2014; 9(2):90083.

Kunkel GT, Maceyka M, Milstien S, Spiegel S. Targeting the sphingosine-1-phosphate axis in cancer, inflammation and beyond. Nat Rev Drug Discov, 2013; 12:688-702.

Lo CG, Xu Y, Proia RL, Cyster JG. Cyclical modulation of sphingosine-1-phosphate receptor 1 surface expression during lymphocyte recirculation and relationship to lymphoid organ transit. J Exp Med, 2005; 201:291-301.

Maruta H, Burgess AW. Regulation of the Ras signalling network. Bioessays, 1994; 16:489-96.

N'Jai AU, Larsen M, Shi L, Jefcoate CR, Czuprynski CJ. Bone marrow lymphoid and myeloid progenitor cells are suppressed in 7,12-dimethylbenz(a)anthracene (DMBA) treated mice. Toxicology, 2010; 271:27-35.

Todd A, Groundwater PW, Gill JH. The global burden of cancer. In: Todd A (eds.). Anticancer therapeutics: from drug discovery to clinical applications. John Wiley \& Sons Ltd., New Jersey, pp. 1-11, 2017.

Niedernberg A, Blaukat A, Schoneberg T, Kostenis E. Regulated and constitutive activation of specific signalling pathways by the human S1PR5 receptor. Br J Pharmacol, 2003; 138:481-93.

Neviani P, Harb JG, Oaks JJ, Santhanam R, Walker CJ, Ellis JJ, Ferenchak G, Dorrance AM, Paisie CA, Eiring AM, Ma Y, Mao HC, Zhang B, Wunderlich M, May PC, Sun C, Saddoughi SA, Bielawski J, Blum W, Klisovic RB, Solt JA, Byrd JC, Volinia S, Cortes J, Huettner CS, Koschmieder S, Holyoake TL, Devine S, Caligiuri MA, Croce CM, Garzon R, Ogretmen B, Arlinghaus RB, Chen C-S, Bittman R, Hokland P, Roy D-C, Milojkovic D, Apperley J, Goldman JM, Reid A, Mulloy JC, Bhatia R, Marcucci G, Perrotti D. PP2A-activating drugs selectively eradicate TKI-resistant chronic myeloid leukemic stem cells. J Clin Invest, 2013; 123:4144-57.

Nishimura H, Akiyama T, Monobe Y, Matsubara K, Igarashi Y, Abe M, Sugihara T, Sadahira Y. Expression of sphingosine-1-phosphate receptor 1 in mantle cell lymphoma. Mod Pathol, 2010; 23:439-49.

Ogretmen B. Sphingolipid metabolism in cancer signalling and therapy. Nat Rev Cancer, 2018; 18:33-50.

Osaka M, Matsuoa S, Koh T, Liang P, Kinoshitaa H, Maedab S, Sugiyama T. N-ras mutation in 7,12-dimethylbenz[a]anthracene (DMBA)induced erythroleukemia in Long-Evans rats. Cancer Lett, 1995; 91:25-31.

Pappu R, Schwab SR, Cornelissen I, Pereira JP, Regard JB, Xu Y, Camerer E, Zheng YW, Huang Y, Cyster JG, Coughlin SR. Promotion of lymphocyte egress into blood and lymph by distinct sources of sphingosine1-phosphate. Science, 2007; 316:295-8.

Pokharel M. Leukemia: a review article. IJARPB, 2012; 2: 397-407. $1618-30$.

Pui C-H. Childhood leukemias. N Engl J Med, 1995; 332

Sessions J. Chronic Myeloid Leukemia in 2007. Am J Health Syst Pharm, 2007; 13:4-7.
Shah MV, Zhang R, Irby R, Kothapalli R, Liu X, Arrington T, Frank B, Lee NH, Loughran TP. Molecular profiling of LGL leukemia reveals role of sphingolipid signaling in survival of cytotoxic lymphocytes. Blood, 2008; 112:770-781.

Siegel RL, Miller KD, Jemal A. Cancer statistics, 2018. CA Cancer J Clin, 2018; 68:7-30.

Subramanian V, Gowry S. Antitumor activity and antioxidant role of Brassica oleracea Italica against Ehrlich ascites carcinoma in swiss albino mice. Res J Pharmaceut Biol Chem Sci, 2011; 2:275-85.

Sugiyama T, Osaka M, Koami K, Maeda S, Ueda N. 7,12-DMBAinduced rat leukemia: a review with insights into future research. Leukoc Res, 2002; 26:1053-68.

Till KJ, Pettitt AR, Slupsky JR. Expression of functional sphingosine-1 phosphate receptor-1 is reduced by B cell receptor signaling and increased by inhibition of PI3 kinase d but not SYK or BTK in chronic lymphocytic leukemia cells. J Immunol, 2015; 194:2439-46.

Unak T, Unak P. Direct radioiodination of metabolic 8-hydroxyquinolyl-glucuronide, as a potential anti-cancer drug. Appl Radiat Isot, 1996; 47:645-7.

Walzer T, Chiossone L, Chaix J, Calver A, Carozzo C, GarrigueAntar L, Jacques Y, Baratin M, Tomasello E, Vivier E. Natural killer cel trafficking in vivo requires a dedicated sphingosine 1-phosphate receptor. Nat Immunol, 2007; 8:1337-44.

Watters RJ, Wang H-G, Sung S-S, Loughran TP, Liu X. Targeting sphingosine-1-phosphate receptors in cancer. Anticancer Agents Med Chem, 2011; 11:810-7.

Zarranz B, Jaso A, Aldana I, Monge AB. Synthesis and anticancer activity evaluation of new 2-alkylcarbonyl and 2-benzoyl-3trifluoromethyl-quinoxaline 1,4-di-N-oxide derivatives. Bioorg Med Chem, 2004; 12:3711-21.

Zhang N, Qi Y, Wadham C, Wang L, Warren A, Di W, Xia P. FTY720 induces necrotic cell death and autophagy in ovarian cancer cells: a protective role of autophagy. Autophagy, 2010; 6:1157-67.

How to cite this article:

Kabeel MM, Ghoneim AM. N-Butylpyridoquinoxaline 1, 4-dioxide alleviates experimentally induced leukemia with up-regulating sphingosine-1-phosphate receptors. J Appl Pharm Sci, 2019; 9(04): 008-015. 